\title{
Implementation of Adaptive Algorithm for PCG Signal Denoising
}

\author{
Mr. R. M. Potdar ${ }^{1}$, Dr. Mekhram Meshram², Naveen Dewangan ${ }^{3}$ \& Dr. Ramesh Kumar ${ }^{4}$ \\ Associate Professor, M. Tech. Coordinator, Electronics \& Telecommunication, BIT, Durg, India ${ }^{1}$ \\ Head of Department, Electronics \& Telecommunication Engineering, GEC, Bilaspur, India ${ }^{2}$ \\ M. Tech. (Scholar), Instrumentation \& Control, BIT, Durg, India ${ }^{3}$ \\ Professor, Computer Science Engineering, BIT, Durg, India ${ }^{4}$
}

\begin{abstract}
Heart sound signals will replicate the physiological and pathological characteristics of the heart. every heart beat is incredibly advanced and short and also the main frequency of heart sound signals is within the range of $10 \mathrm{~Hz}$ to 250Hz. Phonocardiogram will record heart sounds, noise and also the extra sounds. Therefore it's a crucial complement to form up center diagnostic technique examination. Heart sounds are very weak acoustic signals. Within the method to gather heart sound signals it's prone to external acoustic signals and electrical noise interference, especially, the friction caused by subjects respiratory or body movement. The sounds made by friction within the phonocardiogram may produce to an enormous busy signal. Thus, it is vital to research heart sound accurately and eliminates the busy signal with success throughout pre-processing.
\end{abstract}

The objective of this work is to serve as how Noise can be combated using adaptive filter for PCG signal. The problem of controlling the noise level has been one of the research topics over the years. This work focuses on Adaptive filtering algorithms and some of the applications of adaptive filter. The main concept is to use the LMS (Least-Mean-Square) algorithm to develop an adaptive filter that can be used in Adaptive noise Cancellation (ANC) application. In this paper we will learn the various algorithms of LMS (Least Mean Square), NLMS (Normalized Least Mean Square) and RLS (Recursive Least Square) on MATLAB platform with the intention to compare their performance in noise cancellation. The adaptive filter in MATLAB with a noisy tone signal and white noise signal and analyze the performance of algorithms in terms of MSE (Mean Squared Error), percentage noise removal, Signal to Noise Ratio, computational complexity and stability. The Adaptive Filter maximizes the signal to noise ratio \& minimize the Mean Squared Error and compare their performance with respect to stability. Adaptive Noise Canceller is useful to improve the S/N ratio.

This Paper involves the study of the principles of adaptive Noise Cancellation (ANC) and its Applications. Adaptive noise Cancellation is another technique of estimating signals corrupted by additive noise or interference. Its advantage lies in this, with no apriority estimates of signal or noise, levels of noise rejection are attainable that would be difficult or not possible to achieve by other signal processing methods of removing noise. Its cost, inevitably, is that it wants two inputs - a primary input containing the corrupted signal and a reference input containing noise correlate in some unknown approach with the first noise. The reference input is adaptively filtered and subtracted from the first input to get the signal estimate. Adaptive filtering before subtraction permits the treatment of inputs that are settled or random, stationary or time-variable. The result of uncorrelated noises in primary and reference inputs, and presence of signal parts within the reference input on the ANC performance is investigated. it's shown that within the absence of uncorrelated noises and once the reference is free from signal, noise within the primary input can be eliminated while not signal distortion. A configuration of the adaptive noise canceller that does not need a reference input and is incredibly helpful applications is additionally conferred.

Keywords: LMS (Least Mean Square), NLMS (Normalized Least Mean Square), RLS (Recursive Least Square), MSE (Mean Squared Error).

\section{INTRODUCTION}

The noise cancellation technique is a part of optimal these techniques do not cover all the explicit speech filtering that can be applied only when we have models. Each of them is associated with a particular type predetermined knowledge about the reference noise level. of distortion while maximizing noise-reduction effects. Some of its applications are:- speech processing, echo There have been several methods used to study the noise cancellation and enhancement, antenna array processing, cancellation problems. One of the basic and important biomedical signal and image processing and so on. There noise cancellation methods is adaptive filtering. Adaptive are numerous denoising techniques used in speech filters have several applications in acoustics, controls, processing. Most of them include hypotheses on the communications, and coding. Its structure varies from a original signal, as well as snr ratio and distortion. However very simple to complex one. A digital communication 
system consists of a transmitter, channel and receiver connected together. The channel has two major problems, namely, inter symbol interference and noise. The basic principle of noise cancellation is to have an estimate of the interfering signal and subtract it from the corrupted signal.

Adaptive noise cancellation is an interference cancellation technique in itself which relies on the use of noise cancellation by subtracting noise from a received signal, an operation controlled in an adaptive manner for the purpose of improved signal to noise ratio.

\section{Phonocardiogram (PCG): A New Biometric}

A Phonocardiogram or PCG is a plot of high fidelity recording of the sounds and murmurs made by the heart with the help of the machine called phonocardiograph, or "Recording of the sounds made by the heart during a cardiac cycle." Page Layout these sounds are due to vibrations created by closure of the heart valves. There are at least two: the first is when atrio ventricular valves close at the beginning of systole and the second one is when the aortic valve and pulmonary valve close at the end of systole. PCG detects these sub audible sounds and murmurs, and makes a permanent record of these events.

In contrast, the ordinary stethoscope cannot detect such sounds or murmurs, and provides no record of their occurrence. This measurement of the sounds made by the heart provides information not readily available from more sophisticated tests, but at the same time it also provides vital information about the effects of certain cardiac drugs upon the heart. It is also an effective and important method for tracking the progress of the patient's disease.

Heart auscultation is a fundamental tool in the diagnosis of heart diseases. But now a day it has been less focused due to the emergence of ECG and echocardiography; still there are some cardiac defects that are best detected by heart sounds. The human heart is a four-chamber pump with two auricles for the collection of blood from the veins and two ventricles for pumping out the blood to the arteries.

The mechanical functionality of the cardiovascular system is governed by an electrical signal originated in specialized pacemaker cells in the right atrium (the sinoatria node), and is propagated through the atria to the AVnode (a delay junction) and to the ventricles. The periodic beating of the heart is due to complex interaction among pressure gradients, the dynamics of blood flow, and the compliance of cardiac chambers and blood vessels.

The flow of blood is controlled by two sets of valves control: the AV-valves (mitral and tricuspid) between the atria and the ventricles, and the semilunar valves (aortic and pulmonary) between the ventricles and the arteries. These mechanical processes results in vibrations and acoustic signals that can be recorded over the chest wall. The cardiac cycle events are demonstrated in Figure1

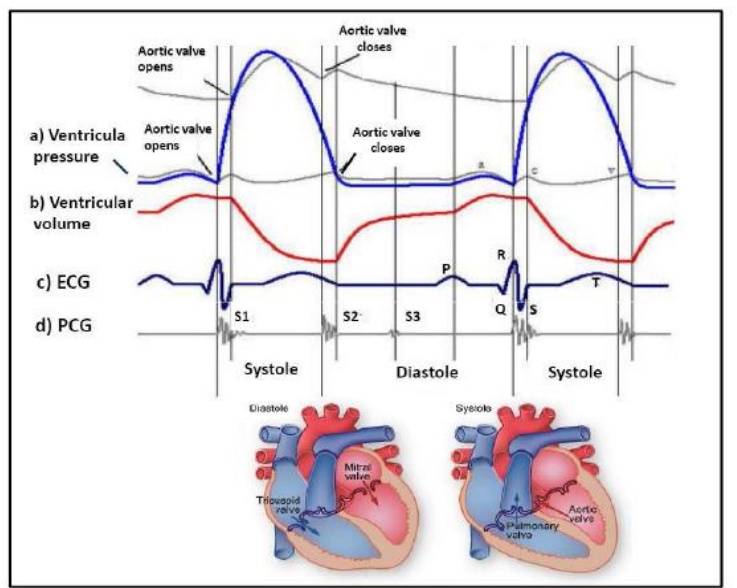

Figure 1: The cardiac cycle, (a) Ventricular Pressure, (b) Ventricular volume, (c) ECG trace, (d) PCG signal

The cardiac cycle consists of two periods, systole and diastole respectively. Both are periods of relatively high activity, alternating with comparatively long intervals of low activity. The major audible components of the PCG are short beats which are recognized as the primary components (S1, S2, S3, and S4). The other classes of sounds are murmurs, clicks, and snaps. However, the two major audible sounds in a normal cardiac cycle are the first and second heart sound, S1 and S2 as depicted in Figure 2.

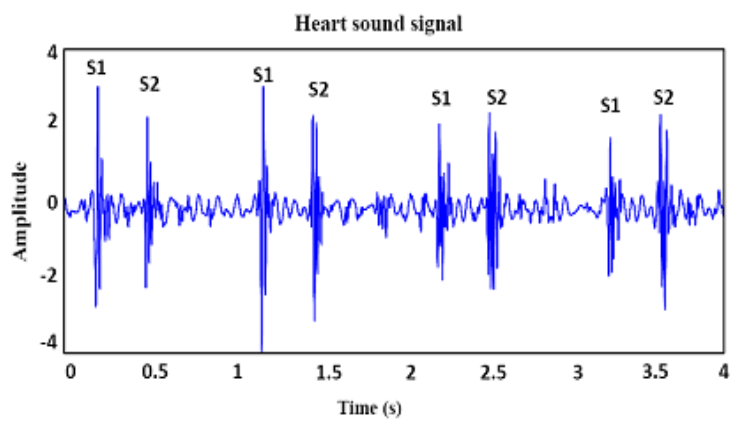

Figure 2: Different components of a normal PCG signal.

- $\mathrm{S}_{1}$ : occurs at the onset of the ventricular contraction during the closure of the $\mathrm{AV}$ - valves. It contains a series of low-frequency vibrations, and is usually the longest and loudest component of the PCG signal. The audible sub-components of $\mathrm{S}_{1}$ are those associated with the closure of each of the two AVvalves. $S_{1}$ lasts for an average period of $100 \mathrm{~ms}-200 \mathrm{~ms}$ and its frequency components lie in the range of $25 \mathrm{~Hz}-45 \mathrm{~Hz}$. It is usually a single component, but may be prominently split with some pathologies.

- $\mathrm{S}_{2}$ : is heard at the end of the ventricular systole, during the closure of the semilunar valves. $\mathrm{S}_{2}$ lasts about $0.12 \mathrm{~s}$, with a frequency of $50 \mathrm{~Hz}$ which is typically higher than $S_{1}$ in terms of frequency content and shorter in terms of duration. It has aortic and pulmonary subcomponents: $\mathrm{A}_{2}$ and $\mathrm{P}_{2}$ corresponding 
to the aortic part and pulmonary part respectively. Usually $A_{2}$ and $P_{2}$ are closed together, but a split $S_{2}$ can occur if $\mathrm{A}_{2}$ and $\mathrm{P}_{2}$ are just far enough apart that they can be heard as two beats within $\mathrm{S}_{2}$.

- $\mathrm{S}_{3}$ : is the third low-frequency sound that may be heard at the beginning of the diastole, during the rapid filling of the ventricles. Its occurrence can be normal in young people (less than 35 years of age).

- S4: is the fourth heart sound that may occur in late diastole during atrial contraction shortly before S1. It is always considered as an abnormality within the cardiac cycle.

- Click and Snaps: are associated with valves opening and indicate abnormalities and heart defects. Opening snaps of the mitral valve or ejection sound of the blood in the aorta may be heard in case of valve disease (stenosis, regurgitation). The most common click is a systolic ejection click, which occurs shortly after $S_{1}$ with the opening of the semilunar valves. The opening snap when present, occurs shortly after $S_{2}$ with the opening of the mitral and tricuspid valves.

- Murmurs: are high-frequency, noise-like sounds that are heard between the two major heart sounds during systole or diastole. They are caused by turbulence in the blood flow through narrow cardiac valves or reflow through the atrioventricular valves due to congenital or acquired defects. They can be innocent, but can also indicate certain cardiovascular defects.

\section{Introduction to Adaptive Filter}

An adaptive filter is a computational device that attempts to model the relationship between two signals in real time in an iterative manner. Adaptive filters are often realized either as a set of program instructions running on an arithmetical processing device such as a microprocessor or DSP chip, or as a set of logic operations implemented in a field-programmable gate array (FPGA) or in a semicustom or custom VLSI integrated circuit. However, ignoring any errors introduced by numerical precision effects in these implementations, the fundamental operation of an adaptive filter can be characterized independently of the specific physical realization that it takes. For this reason, we shall focus on the mathematical forms of adaptive filters as opposed to their specific realizations in software or hardware. Descriptions of adaptive filters as implemented on DSP chips and on a dedicated integrated circuit can be found in (Hanna, 2002; Watrous, 2006; Messer , 2001;), and (Tavel ; 2006), respectively.

An adaptive filter is defined by four aspects:

1) The signals being processed by the filter.

2) The structure that defines how the output signal of the filter is computed from its input signal.

3) The parameters within this structure that can be iteratively changed to alter the filter's input-output relationship.

4) The adaptive algorithm that describes how the parameters are adjusted from one time.
By choosing a particular adaptive filter structure, one specifies the number and type of parameters that can be adjusted. The adaptive algorithm used to update the parameter values of the system can take on a myriad of forms and is often derived as a form of optimization procedure that minimizes an error criterion that is useful for the task at hand.

\section{Active Noise Cancelling}

The active noise cancelling (ANC), also called adaptive noise cancelling or active noise canceller belongs to the interference cancelling class. The aim of this algorithm, as the aim of any adaptive filter, is to minimize the noise interference or, in an optimum situation, cancel that perturbation (Haykin, 1996, Farhang-Boroujeny, 1998, Gale, 2011, Diniz, 2008). The approach adopted in the ANC algorithm, is to try to imitate the original signal $s(n)$. In this study, the final objective is to use an ANC algorithm to cancel speech noise interference, but this algorithm can be employed to deal with any other type of corrupted signal. A scheme of the ANC can be viewed in figure depicted below.

The first work in Adaptive Noise Cancelling was done by two scientists Howells and Applebaum and their colleagues at General Electrical Company. They designed a system for antenna side lobe cancelling that used a reference input derived from an auxiliary antenna and a basic two-weight adaptive filter ${ }^{1}$.

An adaptive filter is a system with a linear filter that has a transfer function controlled by variable parameters and a means to adjust those parameters according to an optimization algorithm. Because of the complex nature of the optimization algorithms, most adaptive filters are digital filters. Adaptive filters are required for some applications because some parameters of the desired processing operation are not known in advance or are changing continuously. The closed loop adaptive filter uses feedback in the form of an error signal to refine its transfer function.

The first adaptive noise cancelling system was designed at Stanford University and built in 1965. And its purpose was to cancel $60 \mathrm{~Hz}$ interference at the output of an electrocardiographic amplifier and recorder $^{1}$.The basic concept of Adaptive noise cancelling is shown in figure.

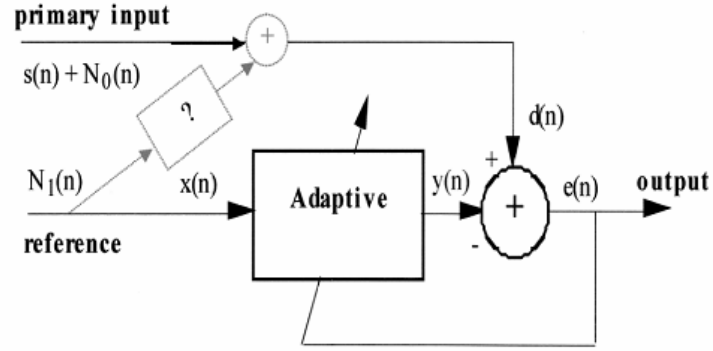

Fig3: Adaptive Noise Canceller
- Source signal

- Primary signal 
INTERNATIONAL JOURNAL OFINNOVATIVE RESEARCH IN ELECTRICAL, ELECTRONICS, INSTRUMENTATION AND CONTROL ENGINEERING Vol. 3, Issue 4, April 2015

$\begin{array}{ll}\mathrm{No}(\mathrm{n}) & - \text { Noise signal } \\ \mathrm{N}_{1}(\mathrm{n}) & - \text { Noise reference input } \\ \mathrm{y}(\mathrm{n}) & - \text { Output of Adaptive Filter } \\ \mathrm{e}(\mathrm{n}) & - \text { System Output Signal }\end{array}$

\subsubsection{Adaptive Filtering}

The main of an adaptive filter in noise cancellation is to remove the noise from a signal adaptively to increase signal to noise (SNR) ratio and finally to improve the quality of signal.

A signal s(n) is transmitted through a channel to a sensor and also receives noise $\operatorname{No}(\mathrm{n})$ uncorrelated with the signal. The combined signal and noise $(s(n)+\operatorname{No}(n))$ form the primary input to the canceller. Now a second sensor receives a noise $\mathrm{N}_{1}(\mathrm{n})$ uncorrelated with the signal but correlated in some unknown way with the noise $\mathrm{No}(\mathrm{n})$. Now this sensor provides the reference input to the canceller. The noise $\mathrm{N}_{1}(\mathrm{n})$ is filtered to produce an output $\mathrm{y}(\mathrm{n})$ that is in resemblance(not exactly) with $\mathrm{No}(\mathrm{n})$.

This output is subtracted from the primary input $[\mathrm{s}(\mathrm{n})+$ $\mathrm{No}(\mathrm{n})]$ to produce the output of the system.

$\mathrm{e}(\mathrm{n})=\mathrm{s}(\mathrm{n})+\operatorname{No}(\mathrm{n})-\mathrm{y}(\mathrm{n})$

The reference input is processed by an adaptive filter, an adaptive filter is a system with a linear filter that has a transfer function controlled by variable parameters and a means to adjust those parameters according to an optimization algorithm. The adaptive filter automatically adjusts its own parameters through an algorithm and responds to an error signal, which depends on the filter's output. In noise cancellation using adaptive filter its objective to produce an error signal that is fit in the least square sense to the signal $s(n)$. This is achieved by feeding back the system output to the adaptive filter using an LMS algorithm to minimize the error signal until it reaches the value $(e(n)=s(n))$

Assume that the $\mathrm{s}(\mathrm{n})$ is the signal which is to be transmitted. $\operatorname{No}(\mathrm{n}), \mathrm{N}_{1}(\mathrm{n})$, and $\mathrm{y}(\mathrm{n})$ are statistically stationary signal. Let assume that $s(n)$ is uncorrelated with $\operatorname{No}(n)$ and $N_{1}(n)$, and $N_{1}(n)$ is correlated with $N o(n)$.

The output $\mathrm{e}(\mathrm{n})$ is given by

$\mathrm{e}(\mathrm{n})=\mathrm{s}(\mathrm{n})+\mathrm{No}(\mathrm{n})-\mathrm{y}(\mathrm{n})$

Taking square both sides,

$\mathrm{e}^{2}(\mathrm{n})=\{\mathrm{s}(\mathrm{n})+\operatorname{No}(\mathrm{n})-\mathrm{y}(\mathrm{n})\}^{2}$

$\mathrm{e}^{2}(\mathrm{n})=\mathrm{s}^{2}(\mathrm{n})+\{\operatorname{No}(\mathrm{n})-\mathrm{y}(\mathrm{n})\}^{2}+2 \mathrm{~s}(\mathrm{n})\{\operatorname{No}(\mathrm{n})-\mathrm{y}(\mathrm{n})\}$

$$
\text { ....... }
$$

Taking the expected value both sides, we get

$\mathrm{E}\left[\mathrm{e}^{2}(\mathrm{n})\right]=\mathrm{E}\left[\mathrm{s}^{2}(\mathrm{n})\right]+\mathrm{E}\left[\{\mathrm{No}(\mathrm{n})-\mathrm{y}(\mathrm{n})\}^{2}\right]+2 \mathrm{E}[\mathrm{s}(\mathrm{n})\{\mathrm{No}(\mathrm{n})$ $-\mathrm{y}(\mathrm{n})\}]$

Realizing that $s(n)$ is uncorrelated with $\mathrm{No}(\mathrm{n})$ and $\mathrm{N}_{1}(\mathrm{n})$ and with $\mathrm{y}(\mathrm{n})$, then

$\mathrm{E}\left[\mathrm{e}^{2}(\mathrm{n})\right]=\mathrm{E}\left[\mathrm{s}^{2}(\mathrm{n})\right]+\mathrm{E}\left[\{\mathrm{No}(\mathrm{n})-\mathrm{y}(\mathrm{n})\}^{2}\right] \quad \ldots \ldots$.

The signal power $E\left[s^{2}(n)\right]$ will be unaffected as the filter is adjusted to minimize $E\left[\mathrm{e}^{2}(\mathrm{n})\right]$. The minimum output power will be given by

$$
\min E\left[e^{2}(n)\right]=E\left[s^{2}(n)\right]+\min E\left[\{\operatorname{No}(n)-y(n)\}^{2}\right]
$$

When the filter is adjusted so that $\mathrm{E}\left[\mathrm{e}^{2}(\mathrm{n})\right]$ is minimized, $\mathrm{E}\left[\{\operatorname{No}(\mathrm{n})-\mathrm{y}(\mathrm{n})\}^{2}\right]$ is also minimized. When $\mathrm{E}[\{\mathrm{No}(\mathrm{n})-$ $\left.\mathrm{y}(\mathrm{n})\}^{2}\right]$ is minimized then $\mathrm{E}\left[\{\mathrm{e}(\mathrm{n})-\mathrm{s}(\mathrm{n})\}^{2}\right]$ is also minimized, and hence

$[\mathrm{e}(\mathrm{n})-\mathrm{s}(\mathrm{n})] \approx[\mathrm{No}(\mathrm{n})-\mathrm{y}(\mathrm{n})]$

Here, The output e(n) will contain the signal s(n) and noise. From (1), the output noise is given by [ No(n) $y(n)]$. Since minimizing $E\left[e^{2}(n)\right]$ minimizes $E[\{N o(n)-$ $\left.y(n)\}^{2}\right]$, and since the signal in the output remains constant, minimizing the total output power maximizes the output signal-to-noise ratio. From (3), it seems that the smallest possible output power is $\mathrm{E}\left[\mathrm{e}^{2}(\mathrm{n})\right]=\mathrm{E}\left[\mathrm{s}^{2}(\mathrm{n})\right]$ and that will lead to $\mathrm{E}\left[\{\mathrm{No}(\mathrm{n})-\mathrm{y}(\mathrm{n})\}^{2}\right] \approx 0$. Therefore $y(n)=\operatorname{No}(n)$, and $e(n)=s(n)$

Hence minimizing output power causes the output signal to be noise free.

\section{Literature Review:}

Adaptive equalization of telephone channels to minimize data transmission intersymbol interference was first developed by Lucky in 1965 (Lucky 1965). He used his minimax criterion based zero-forcing algorithm to automatically adjust the tap weights of a transversal equalizer by minimizing what he called the peak distortion. This pioneering work by Lucky spearheaded many other significant contributions to the adaptive equalization problem. In 1969, Gerosho and Proakis, and Miller independently reformulated the adaptive equalizer problem using a mean square-error criterion. In 1978 Falconer and Ljung (Falconer and Ljung 1978) developed a simplifying modification to a Kalman based algorithm, for adaptive tap adjustment, derived by Godard in 1974 . This simplification reduced the computational complexity of Godard's algorithm to that comparable with the LMS algorithm. Satorius, Alexander and Pack in the late 1970s and early $80 \mathrm{~s}$ showed the usefulness of lattice-based algorithms for adaptive equalization.

Linear Predictive Coding (LPC) was introduced and developed for the problem of speech coding in the early

(1) $1970 \mathrm{~s}$ by Atal and Hanauer. In LPC the speech waveform is represented directly in terms of time-varying parameters related to the transfer function of the vocal tract and excitation characteristics. The predictor coefficients are 2) determined by minimizing the mean square error between actual and predicted speech samples. Although a lattice structure for the linear prediction problem was developed by a number of investigators it was Saito and Itakura who were credited with the invention in 1972. They were able to show that the filtering process of a lattice predictor model and an acoustic tube model of speech were identical. From the time when Schuster invented the periodogram for analyzing the power spectrum of a timeseries in 1898 until 1927 it was the only numerical method available for spectrum analysis. In 1927 Yule (Yule 1927) introduced a new approach based on the concept of a finite 
parameter model for a stationary stochastic process. This new approach was developed to combat the problem of the periodogram's erratic behaviour when applied to empirical time-series observed in nature such as sunspot activity. Yule's model was a stochastic feedback model in which the present sample of the time-series is assumed to consist of a linear combination of past samples plus an error term. This approach was called autoregressive spectrum analysis. Burg rekindled interest in the autoregressive method in the 1960s and 70s with his maximum-entropy method of power spectrum estimation directly from the available time-series.

In 1971 Van den Bos (Van den Bos 1971) was able to show that the maximum-entropy method is equivalent to least squares fitting of an autoregressive model to the known autocorrelation sequence. The maximum-entropy method involved the extrapolation of the autocorrelation function of the time series in such a way that the entropy of the corresponding probability is maximized at each step of the extrapolation. In 1967 Kelly of Bell Telephone Laboratories was given credit for inventing an adaptive filter for speech echo cancellation, which used the speech signal itself in the adaptation processes. Work on echo cancellers only started around 1965. Another type of adaptive noise canceller was the line canceller used for removing the mains power frequency interference from instrument and sensor preamplifier circuits. This was invented by Widrow and his co-workers at Stanford University. An early version of the device was built in 1965 and described in Widrow's paper in 1975 (Widrow et al 1975).

Initial contributions to adaptive array antennas were made by Howells in the late 1950s and by Applebaum in 1966. Howells developed a sidelobe canceller that became a special case of Applebaum's adaptive antenna array system. Applebaum's algorithm was based on maximizing the Signal-to-Noise Ratio (SNR) at the array output for any type of noise. This classic work was reprinted in the 1976 special issue of IEEE Transactions on Antennas and Propagation (Applebaum and Chapman 1976). Another major work related to adaptive array antennas was put forward independently by Widrow and his co-workers in 1967. Their theory was based on the LMS algorithm and their paper, (Widrow et al 1967), the first publication in the open literature on adaptive array antenna systems, was considered to be another classic of that era. In 1969 Capon (Capon 1969) proposed a different method for solving the adaptive beam forming problem based on variance (average power) minimization. Finally, in 1983, McWhirter (McWhirter 1983) developed a simplified version of the Gentleman-Kung systolic array for recursive least squares estimation, which is very well suited for adaptive beam forming applications.

\section{Problem Identification in PCG Signal}

Auscultation, the noninvasive cardiac testing, is used as a primary detection tool for diagnosis of heart valve disorders since invention of stethoscope in 1816 by Lannec (Hanna \& Silverman, 2002). Heart sounds provide valuable diagnostic and prognostic information concerning the heart valves and hemodynamic of heart. During the last few decades, valvular heart diseases remain one of the major health concerns. Hence, early detection of heart valve diseases and accurate diagnosis of related conditions comprise a significant medical research area. In Bender (1992), it is reported that few heart valve diseases are best detected only by means of auscultation process. Auscultation is the most common and cost-effective technique, continues to provide an important source of clinical information related to heart valves and also cannot be totally replaced by alternative technical methods like echocardiography (Tavel, 1996). Moreover, echocardiography is not required for all patients with systolic murmurs (Shub, 2003; Tavel, 1996). Chizner (2008) has shown in his practical clinical overview of a variety of cardiac disease states and conditions that the stethoscope often enables many well-trained and experienced cardiac auscultators to make a rapid and accurate cardiac diagnosis. Physicians use the stethoscope as a device to listen the function of heart valves and make a diagnosis accordingly.

However, in studying the physical characteristics of heart sounds and human hearing, it is seen that the human ear is poorly suited for cardiac auscultation (Mangione \& Nieman, 1997). Therefore, relative and qualitative nature of heart sounds is limited by human perception and varies with personal aptitude and training. Phonocardiogram (PCG), a visual display of the recorded heart sounds (Durand \& Pibarot, 1995; Lukkarinen, Nopanen, Sikio, \& Angerla, 1997) provides a trace of acoustic energy produced by the mechanical activity of various cardiac components and processes (Durand \& Pibarot, 1995). Consequently, any abnormality of heart valves is reflected in the corresponding sounds of PCG signal. A cardiac cycle of a normal heart is comprised of two major sounds namely first heart sound, S1 followed by second heart sound, S2. These two distinct normal heart sounds are often described as lub (or lup) and dub (or dup), and occur in sequence with each heart beat. In case of abnormal heart sounds there could be several other sounds in the PCG signal besides primary heart sounds. Murmurs are abnormal heart sounds and refer to different pathological conditions (Cromwell, Weibell, \& Pfeiffer, 2002,) as per location, shape, duration and other associated features.

Murmurs are generally high-frequency, noise like sounds that are produced as a result of turbulent blood flow. Different features of PCG signals like intensity, frequency content, split information, time relations etc. Are helpful in detecting heart valve diseases, if any and the state of the heart function (Rangayyan, 2002). Analysis of phonocardiogram signal can also be carried out by considering the heart sound cycle as a whole instead of separating the major components. Most of the heart sound classification techniques reported so far follows the same line of thought (Ari \& Saha, 2008, 2009; Cathers, 1995; Gupta, Palaniappan, Swaninathan, \& Krishnan, 2007; Ölmez \& Dokur, 2003; Reed, Reed, \& Fritzson, 2004). Time-frequency analysis techniques like wavelet 
transform have been widely used (Ari \& Saha, 2009; Cathers, 1995; Gupta et al., 2007; Ölmez \& Dokur, 2003; Reed et al., 2004) for extracting feature vectors from heart sound signal because of its ability to characterize timefrequency information which is important in this context (Debbal \& Bereksi Reguig, 2007; El-Asir, Khadra, AlAbbasi, \& Mohammed, 1996; Lee, Lee, Kim, Min, \& Hong, 1999; Wood \& Barry, 1995).

\section{Methodology:}

The idea behind a closed loop adaptive filter is that a variable filter is adjusted until the error (the difference between the filter output and the desired signal) is minimized. The Least Mean Squares (LMS) filter, Normalized Least Mean Square (NLMS) filter and the Recursive Least Squares (RLS) filter are types of adaptive filter. All adaptive algorithms LMS has probably become the most popular for its robustness, good tracking capabilities.

\section{LMS algorithm:}

An adaptive filter is a computational device that iteratively models the relationship between the input and output signals of a filter. An adaptive filter self-adjusts the filter coefficients according to an adaptive algorithm. Figure 4 shows the diagram of a typical adaptive filter.

The LMS is one of the simplest algorithms used in the adaptive structures due to the fact that it uses the error signal to calculate the filter coefficients. The output $y(n)$ of FIR filter structure can be obtain from Eq.

$\mathrm{y}(\mathrm{n})=\sum_{m=0}^{N-1} w(m) x(n-m)$

Where $\mathrm{n}$ is no. of iteration

The error signal is calculated by Eq. (2)

$\mathrm{e}(\mathrm{n})=\mathrm{d}(\mathrm{n})-\mathrm{y}(\mathrm{n})$

The filter weights are updated from the error signal e(n) and input signal $\mathrm{x}(\mathrm{n})$ as in Eq. (3).

$\mathrm{w}(\mathrm{n}+1)=\mathrm{w}(\mathrm{n})+\mu \mathrm{e}(\mathrm{n}) x(\mathrm{n})$

Where: $w(n)$ is the current weight value vector, $w(n+1)$ is the next weight value vector, $x(n)$ is the input signal vector, $e(n)$ is the filter error vector and $\mu$ is the convergence factor which determine the filter convergence speed and overall behavior.

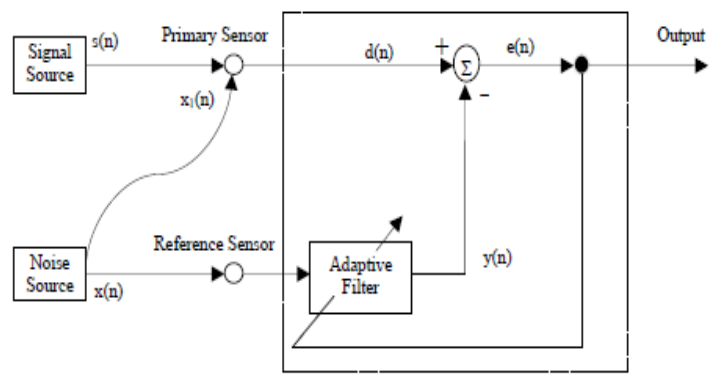

Adaptive Noise Canceller

Fig 4: Adaptive Noise Canceller

$s(n)$

- Source signal

$\mathrm{d}(\mathrm{n})$
$\mathrm{X}_{1}(\mathrm{n}) \quad-$ Noise signal

$\mathrm{X}(\mathrm{n} \quad-$ Noise reference input

$\mathrm{y}(\mathrm{n}) \quad-$ Output of Adaptive Filter

e(n) $\quad-$ System Output Signal

\section{NLMS Algorithm:}

In the standard LMS algorithm, when the convergence factor $\mu$ is large, the algorithm experiences a gradient noise amplification problem. In order to solve this difficulty, we can use the NLMS (Normalized Least Mean Square) algorithm. The correction applied to the weight vector $w(n)$ at iteration $n+1$ is "normalized" with respect to the squared Euclidian norm of the input vector $\mathrm{x}(\mathrm{n})$ at iteration $n$. We may view the NLMS algorithm as a timevarying step-size algorithm, calculating the convergence factor $\mu$ as in Eq. (4)[7].

$\mu(n)=\frac{\alpha}{c+(\|x(n)\|)^{2}}$

Where: $\alpha$ is the NLMS adaption constant, which optimize the convergence rate of the algorithm and should satisfy the condition $0<\alpha<2$, and $\mathrm{c}$ is the constant term for normalization and is always less than 1 . The Filter weights are updated by the Eq. (5).

$\mathrm{w}(\mathrm{n}+1)=\mathrm{w}(\mathrm{n})+\frac{\alpha}{c+\left(\|x(n)\|^{2}\right.} \mathrm{e}(\mathrm{n}) x(\mathrm{n})$

\section{RLS Algorithm:}

The RLS algorithms are known for their excellent performance when working in time varying environments but at the cost of an increased computational complexity and some stability problems. In this algorithm the filter tap weight vector is updated using Eq. (6) [8].

$\mathrm{w}(\mathrm{n})=\bar{w}^{T}(\mathrm{n}-1)+\mathrm{k}(\mathrm{n}) \bar{e}_{n-1}(n)$

Eq. (7) and (8) are intermediate gain vector used to compute tap weights.

$\mathrm{k}(\mathrm{n})=\mathrm{u}(\mathrm{n}) /\left[\lambda+x^{T}(n) u(n)\right]$

$\mathrm{u}(\mathrm{n})=\overline{w_{\lambda}^{-1}}(\mathrm{n}-1) x(\mathrm{n})$

Where: $\lambda$ is a small positive constant very close to, but smaller than 1 .

The filter output is calculated using the filter tap weights of previous iteration and the current input vector as in Eq. (9).

$\bar{y}_{n-1}(n)=\bar{w}^{T}(\mathrm{n}-1) x(\mathrm{n})$
$\bar{e}_{n-1}(n)=\mathrm{d}(\mathrm{n})-\bar{y}_{n-1}(n)$.

In the RLS Algorithm the estimate of previous samples of output signal, error signal and filter weight is required that leads to higher memory requirements.

\section{Result:}

Table 5.1: Result of different Adaptive Filter Algorithms at different Cut Off frequency with filter order $=31 \&$ Step Size $=.08$

Note: Input signal length $=10000$

Filter Order $=31 \quad ;$ Step Size $=0.08$ 
INTERNATIONAL JOURNAL OF INNOVATIVE RESEARCH IN ELECTRICAL, ELECTRONICS, INSTRUMENTATION AND CONTROL ENGINEERING Vol. 3, Issue 4, April 2015

\begin{tabular}{|c|c|c|c|c|c|c|}
\hline \multirow[b]{2}{*}{$\begin{array}{l}\text { S. } \\
\text { No }\end{array}$} & \multirow[b]{2}{*}{$\begin{array}{l}\text { Adapt } \\
\text { ive } \\
\text { Filter } \\
\text { Algor } \\
\text { ithms }\end{array}$} & \multirow[b]{2}{*}{$\begin{array}{c}\text { Cut } \\
\text { Off } \\
\text { freq } \\
\text { uenc } \\
y \\
\end{array}$} & \multicolumn{2}{|c|}{ Testh1 } & \multicolumn{2}{|c|}{ Testh2 } \\
\hline & & & SNR & MSE & $\begin{array}{c}\text { SN } \\
\text { R }\end{array}$ & $\begin{array}{c}\text { MS } \\
\mathbf{E}\end{array}$ \\
\hline \multirow{5}{*}{1} & \multirow{5}{*}{ LMS } & 0.1 & 1.2991 & $\begin{array}{c}2.138 \\
8 \mathrm{e}- \\
006\end{array}$ & $\begin{array}{l}10.9 \\
003\end{array}$ & $\begin{array}{l}2.27 \\
62 \mathrm{e}- \\
008\end{array}$ \\
\hline & & 0.5 & 1.4899 & $\begin{array}{c}4.647 \\
3 \mathrm{e}- \\
007\end{array}$ & $\begin{array}{l}11.3 \\
480\end{array}$ & $\begin{array}{c}2.25 \\
95 \mathrm{e}- \\
008\end{array}$ \\
\hline & & 0.9 & 1.3052 & $\begin{array}{c}1.127 \\
6 \mathrm{e}- \\
006\end{array}$ & $\begin{array}{l}10.8 \\
433\end{array}$ & $\begin{array}{l}1.06 \\
18 \mathrm{e}- \\
007\end{array}$ \\
\hline & & 0.95 & 1.2069 & $\begin{array}{c}3.371 \\
2 \mathrm{e}- \\
006\end{array}$ & $\begin{array}{l}11.0 \\
483\end{array}$ & $\begin{array}{c}2.03 \\
15 \mathrm{e}- \\
008\end{array}$ \\
\hline & & 0.99 & 1.3693 & $\begin{array}{c}1.798 \\
4 \mathrm{e}- \\
006\end{array}$ & $\begin{array}{l}10.6 \\
687\end{array}$ & $\begin{array}{c}9.83 \\
28 \mathrm{e}- \\
009\end{array}$ \\
\hline & & & & & & \\
\hline \multirow{5}{*}{2} & \multirow{5}{*}{$\begin{array}{c}\text { NLM } \\
\mathrm{S}\end{array}$} & 0.1 & 1.1784 & $\begin{array}{c}2.230 \\
9 \mathrm{e}- \\
007\end{array}$ & $\begin{array}{c}7.43 \\
47\end{array}$ & $\begin{array}{c}1.84 \\
60 \mathrm{e}- \\
007 \\
\end{array}$ \\
\hline & & 0.5 & 1.2176 & $\begin{array}{c}8.342 \\
7 \mathrm{e}- \\
007\end{array}$ & $\begin{array}{c}7.40 \\
37\end{array}$ & $\begin{array}{c}8.15 \\
08 \mathrm{e}- \\
007\end{array}$ \\
\hline & & 0.9 & 1.3473 & $\begin{array}{c}4.510 \\
9 \mathrm{e}- \\
006 \\
\end{array}$ & $\begin{array}{c}7.06 \\
10\end{array}$ & $\begin{array}{c}7.43 \\
22 \mathrm{e}- \\
007 \\
\end{array}$ \\
\hline & & 0.95 & 1.1649 & $\begin{array}{c}1.787 \\
3 \mathrm{e}- \\
006\end{array}$ & $\begin{array}{c}7.23 \\
28\end{array}$ & $\begin{array}{c}2.29 \\
48 \mathrm{e}- \\
006\end{array}$ \\
\hline & & 0.99 & 1.0053 & $\begin{array}{c}2.492 \\
7 \mathrm{e}- \\
006 \\
\end{array}$ & $\begin{array}{c}7.36 \\
07\end{array}$ & $\begin{array}{l}7.95 \\
51 \mathrm{e}- \\
007 \\
\end{array}$ \\
\hline & & & & & & \\
\hline \multirow{5}{*}{3} & \multirow{5}{*}{$\begin{array}{c}\text { DLM } \\
\mathrm{S}\end{array}$} & 0.1 & 1.3786 & $\begin{array}{c}7.403 \\
9 \mathrm{e}- \\
007 \\
\end{array}$ & $\begin{array}{l}11.0 \\
987\end{array}$ & $\begin{array}{c}1.18 \\
95 \mathrm{e}- \\
008 \\
\end{array}$ \\
\hline & & 0.5 & 1.3001 & $\begin{array}{c}2.325 \\
0 \mathrm{e}- \\
006 \\
\end{array}$ & $\begin{array}{l}10.4 \\
702\end{array}$ & $\begin{array}{c}8.49 \\
31 \mathrm{e}- \\
009 \\
\end{array}$ \\
\hline & & 0.9 & 1.5178 & $\begin{array}{c}1.483 \\
3 \mathrm{e}- \\
006 \\
\end{array}$ & $\begin{array}{l}10.7 \\
560\end{array}$ & $\begin{array}{c}7.34 \\
09 \mathrm{e}- \\
008 \\
\end{array}$ \\
\hline & & 0.95 & 1.4159 & $\begin{array}{c}1.519 \\
5 \mathrm{e}- \\
007 \\
\end{array}$ & $\begin{array}{l}11.1 \\
030\end{array}$ & $\begin{array}{c}2.01 \\
61 \mathrm{e}- \\
008 \\
\end{array}$ \\
\hline & & 0.99 & 1.5055 & $\begin{array}{c}1.812 \\
7 \mathrm{e}- \\
006 \\
\end{array}$ & $\begin{array}{l}11.4 \\
808\end{array}$ & $\begin{array}{c}9.87 \\
83 \mathrm{e}- \\
008 \\
\end{array}$ \\
\hline & & & & & & \\
\hline \multirow{3}{*}{4} & \multirow{3}{*}{ RLS } & 0.1 & & & - & - \\
\hline & & 0.5 & 0.0043 & $\begin{array}{c}7.284 \\
5 \mathrm{e}- \\
005\end{array}$ & - & - \\
\hline & & 0.9 & 0.8473 & $\begin{array}{c}1.670 \\
1 \mathrm{e}-\end{array}$ & - & - \\
\hline
\end{tabular}

\begin{tabular}{|c|c|c|c|c|c|c|}
\hline & & & & 006 & & \\
\hline & & 0.95 & 1.5312 & $\begin{array}{c}3.366 \\
7 \mathrm{e}- \\
006\end{array}$ & - & - \\
\hline & & 0.99 & $\begin{array}{c}20.698 \\
9\end{array}$ & $\begin{array}{c}4.863 \\
5 \mathrm{e}- \\
007\end{array}$ & - & - \\
\hline & & & & & & \\
\hline \multirow{5}{*}{5} & \multirow{5}{*}{$\begin{array}{l}\text { QRD- } \\
\text { RLS }\end{array}$} & 0.1 & $\begin{array}{c}1.4790 \\
\text { e-004 }\end{array}$ & $\begin{array}{c}0.005 \\
8\end{array}$ & $\begin{array}{l}2.09 \\
69 \mathrm{e}- \\
007\end{array}$ & $\begin{array}{c}5.07 \\
28 \mathrm{e}+ \\
004\end{array}$ \\
\hline & & 0.5 & 0.0036 & $\begin{array}{c}5.825 \\
1 \mathrm{e}- \\
007\end{array}$ & $\begin{array}{l}2.09 \\
15 \mathrm{e}- \\
007\end{array}$ & $\begin{array}{c}5.49 \\
23 \mathrm{e}+ \\
004\end{array}$ \\
\hline & & 0.9 & 0.0014 & $\begin{array}{c}0.002 \\
9\end{array}$ & $\begin{array}{c}7.51 \\
98 \mathrm{e}- \\
006\end{array}$ & $\begin{array}{l}461 . \\
0261\end{array}$ \\
\hline & & 0.95 & $\begin{array}{c}3.1385 \\
\text { e-004 }\end{array}$ & $\begin{array}{c}5.061 \\
3 \mathrm{e}- \\
006\end{array}$ & $\begin{array}{l}1.37 \\
56 \mathrm{e}- \\
007\end{array}$ & $\begin{array}{c}7.09 \\
34 \mathrm{e}+ \\
004\end{array}$ \\
\hline & & 0.99 & 0.0025 & $\begin{array}{c}0.010 \\
9\end{array}$ & $\begin{array}{l}1.64 \\
33 \mathrm{e}- \\
007\end{array}$ & $\begin{array}{c}5.50 \\
80 \mathrm{e}+ \\
004\end{array}$ \\
\hline
\end{tabular}

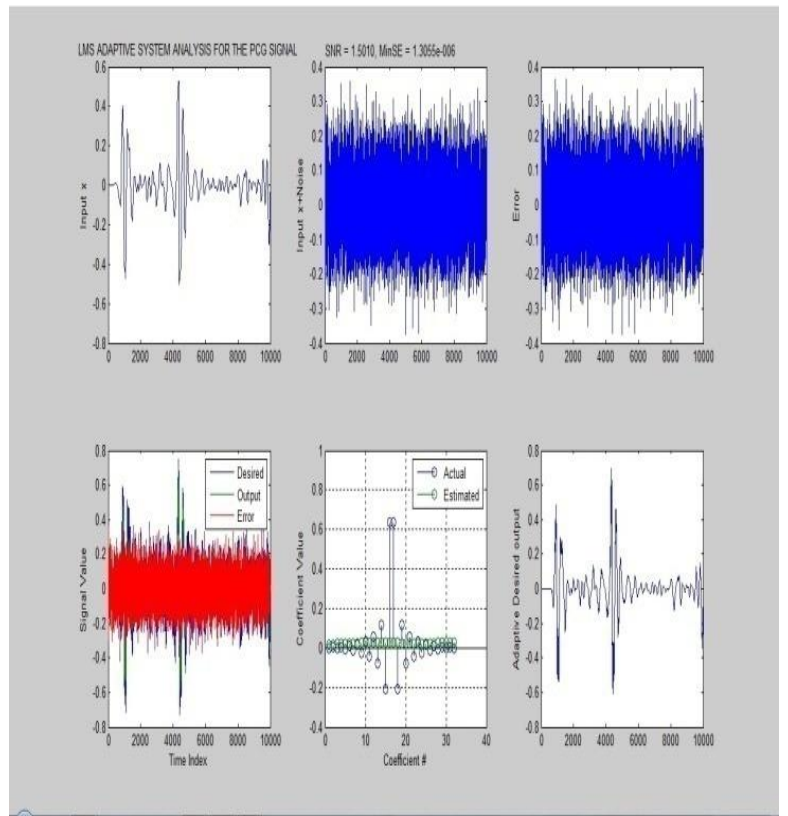

Fig 5.1: LMS ADAPTIVE SYSTEM ANALYSIS FOR THE PCG SIGNAL (Testh1)

Filter Order $=31 \quad$; Cut Off frequency $=0.99$

; Step Size $=0.08$

Above Figure 5.1 represent the LMS Adaptive System Analysis for the PCG Signal (Testh1) in which Filter Order $=31$, Cut Off frequency $=0.99$, Step Size $=0.08$ and LMS Adaptive filter gives SNR $=1.5010$ and $\mathrm{MSE}=$ $1.3055 \mathrm{e}-006$. 
INTERNATIONAL JOURNAL OF INNOVATIVE RESEARCH IN ELECTRICAL, ELECTRONICS, INSTRUMENTATION AND CONTROL ENGINEERING Vol. 3, Issue 4, April 2015

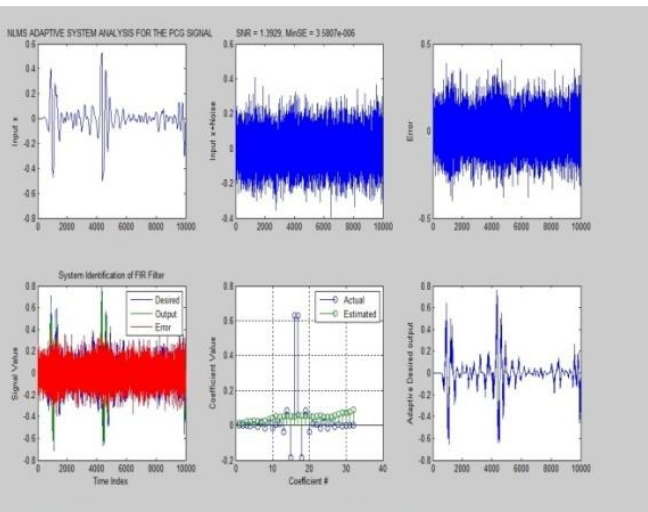

Fig5.2: NLMS ADAPTIVE SYSTEM ANALYSIS FOR THE PCG SIGNAL (Testh1)

Filter Order $=31$; Cut Off frequency $=0.9$; Step Size $=$ 0.99

Above Figure 5.2 represent the NLMS Adaptive System Analysis for the PCG Signal (Testh1) in which Filter Order $=31$, Cut Off frequency $=0.9$, Step Size $=0.99$ and NLMS Adaptive filter gives SNR = 1.3929 and MSE = 3.5807e-006.

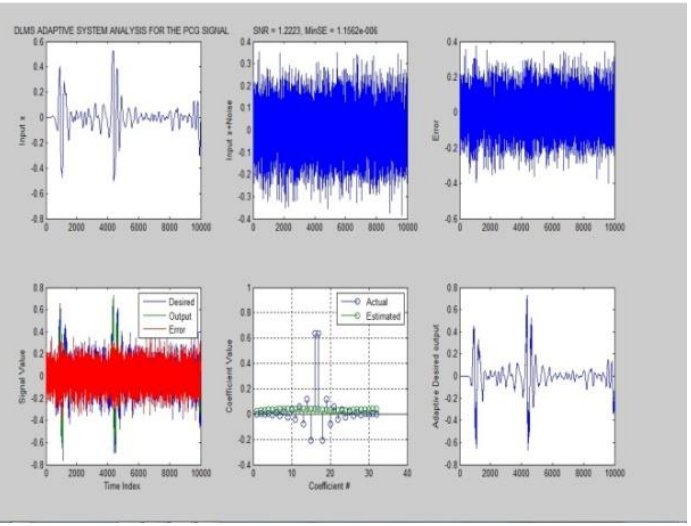

Fig5.3: DLMS ADAPTIVE SYSTEM ANALYSIS FOR THE PCG SIGNAL (Testh1)

Filter Order $=3$; Cut Off frequency $=0.99$; Step Size $=$ 0.08

Above Figure 5.3 represent the DLMS Adaptive System Analysis for the PCG Signal (Testh1) in which Filter Order $=31$, Cut Off frequency $=0.08$, Step Size $=0.08$ and DLMS Adaptive filter gives SNR $=1.2223$ and $\mathrm{MSE}=$ $1.1562 \mathrm{e}-006$.

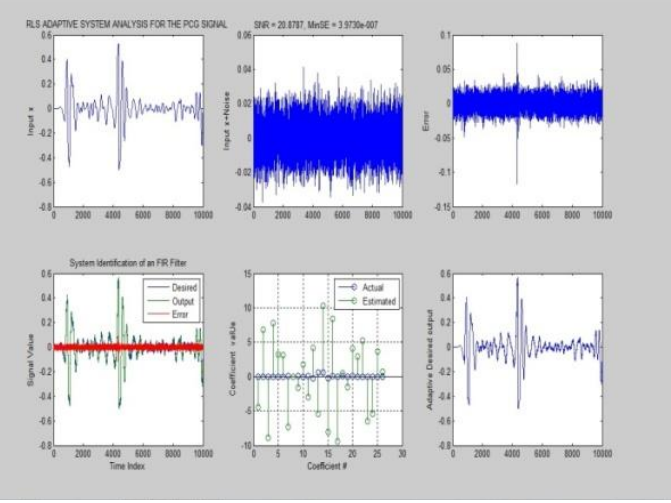

Fig 5.4: RLS ADAPTIVE SYSTEM ANALYSIS FOR THE PCG SIGNAL (Testh1)
Filter Order $=25 \quad$; Cut Off frequency $=0.99 \quad$; Step

Size $=0.99$

Above Figure 5.4 represent the RLS Adaptive System Analysis for the PCG Signal (Testh1) in which Filter Order $=25$, Cut Off frequency $=0.99$, Step Size $=0.99$ and RLS Adaptive filter gives $\mathrm{SNR}=20.8787$ and $\mathrm{MSE}=$ 3.9730e-007.

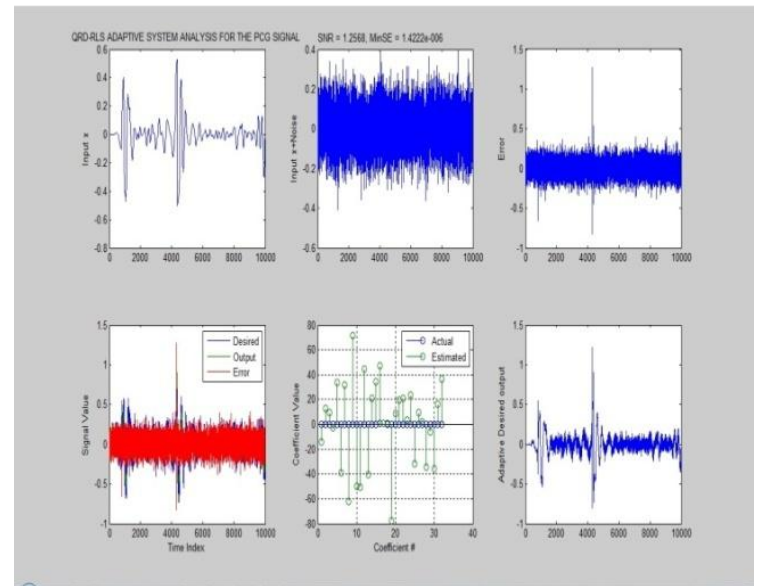

Fig 5.5: QRDRLS ADAPTIVE SYSTEM ANALYSIS FOR THE PCG SIGNAL (Testh1)

Filter Order $=31 \quad ;$ Cut Off frequency $=0.99$

Step Size $=0.99$

Above Figure Fig 5.5 represent the QRDRLS Adaptive System Analysis for the PCG Signal (Testh1) in which Filter Order $=31$, Cut Off frequency $=0.99$, Step Size $=$ 0.99 and QRDRLS Adaptive filter gives SNR = 1.2568 and MSE $=1.4222 \mathrm{e}-006$

\section{CONCLUSION}

In this work, different Adaptive algorithms were analysed and compared. The basic adaptive algorithms which widely used for performing weight updating of an adaptive filter are: the LMS (Least Mean Square), NLMS (Normalized Least Mean Square) and the RLS (Recursive Least Square) algorithm.

Among all adaptive algorithms LMS has probably become the most popular for its robustness, good tracking capabilities and simplicity in stationary environment. RLS is best for non-stationary environment with high convergence speed but at the cost of higher complexity.

The main concept is to use the LMS (Least-Mean-Square) algorithm to develop an adaptive filter that can be used in Adaptive noise Cancellation (ANC) application. In this paper we will learn the various algorithms of LMS (Least Mean Square), NLMS (Normalized Least Mean Square) and RLS (Recursive Least Square) on MATLAB platform with the intention to compare their performance in noise cancellation.

In real-time applications, it is very important to analyses all the important details before we choose an adaptive algorithm. A small difference could result in 
elevated cost of implementation, or in a weak system, When input is non-stationary in nature, the which is not stable in all variable changes, or even the RLS formula proved to possess the best convergence solution is impossible to be implemented. The choice speed, less MSE, and highest proportion of noise between using one algorithm instead of another, to the reduction however at the price of huge process quality and system identification problem, depends mainly on the memory demand.

following factors:

In this research work this two factor has been analyses.

The recursive least squares (RLS) algorithms have a quicker convergence speed and within the derivation of

1) Signal to Noise Ratio (SNR) - The Adaptive Filter maximizes the signal to noise ratio. Adaptive Noise Canceller is useful to improve the $\mathrm{S} / \mathrm{N}$ ratio.

RLS algorithm, the input signals are considered deterministic, whereas for the LMS and different similar algorithmic program they're thought of random.

2) Mean Squared Error (MSE) - The Adaptive Filter minimizes the Mean Squared Error and compare their performance with respect to stability.

However, RLS algorithmic programs involve additional sophisticated mathematical operations and need additional machine resources than the LMS algorithm.

The Adaptive Filter minimizes the mean squared error between a primary input, which is the noisy PCG, and a reference input, which is either noise that is correlated in some way with the noise in the primary input or a signal that is correlated only with PCG in the primary input.

\section{LMS (Least-Mean-Square)}

The main concept is to use the LMS (Least-Mean-Square) algorithm to develop an adaptive filter that can be used in Adaptive noise Cancellation (ANC) application. Among all adaptive algorithms LMS has probably become the most popular for its robustness, good tracking capabilities and simplicity in stationary environment.

The conclusion of on top of results is that, If LMS filter is employed for noise cancellation and quality reduction are main criteria, and if the "Step size $(\mu)$ " is enlarged, LMS algorithmic rule converges a lot of quickly, however at the expense of granularity - the LMS Filter Output isn't as smooth.

Step size isn't too tiny (large) it takes time to converge, and step size isn't large, filter response isn't joining just in case of enormous step size.

Advantages \& disadvantages of LMS algorithm:

1) Simplicity in implementation

2) Stable and robust performance against different signal conditions.

3) slow convergence (due to Eigen value spread)

\section{NLMS (Normalized Least Mean Square)}

NLMS formula changes the step-size in step with the energy of input signals therefore it's appropriate for each stationary in addition as non-stationary setting and its performance lies between LMS and RLS. Therefore it provides a trade-off in convergence speed and process quality. The implementation of algorithms was with success achieved, with results that have a extremely sensible response.

\section{RLS (Recursive Least Square)}

RLS is best for non-stationary environment with high convergence speed but at the cost of higher complexity.

\section{DLMS (Delayed Least Mean Square)}

Delayed LMS algorithm is suited in hard ware implementation with performance degradation. This report introduces three sorts of DLMS formula and its design. DLMS formula has the worst performance however least hardware value. DLMS with correction formula has performance almost like LMS formula however value concerning 2.6 times space than DLMS;

Threshold DLMS with correction formula has nearly a similar space value of DLMS with formula correction however has less power consumption than it.

\section{QRD-RLS (Quadrature Recursive Least Square)}

The QRD-RLS adaptive filters will become attractive in device, sonar, and mobile/wireless communication systems as a result of their superior convergence rate and increasing chip capability.

However, it's still a challenge to style ASIC chip design for such applications as a result of their beat array implementations are of enormous size. The results give a guide to style a fixed-point implementation of QRD RLS adaptive filter.

\section{Scope for future work:}

1) Based upon adaptive algorithms in future we can make intelligent adaptive filter, which measure the quantity of noise in the signal and according to quantity of noise it select different adaptive algorithms like LMS (Least Mean Square), NLMS (Normalized Least Mean Square) and the RLS (Recursive Least Square) algorithm, which will suitable for the signal.

2) Future work can apply method in hardware. Recording LS and HS simultaneously from chest uses two sensors, one for LS and another for HS. The two recording signals will be applied to inputs of LMSANC.

This trend can be applied in clinical diagnoses for HS and LS. It requires the development of instruments and data analysis. 


\section{REFERENCES}

[1] R. M. Potdar, Tripti Roy / International Journal of Engineering and Advanced Technology (IJEAT) ISSN: 2249 - 8958, Volume-1, Issue-3, February 2012. "Performance Evaluation of Different Adaptive Filtering Algorithms for Reduction of Heart Sound from Lung Sound"

[2] R. M. Potdar, Nishi Shahnaj Haider / International Journal of Engineering Research and Applications (IJERA) ISSN: 2248-9622 www.ijera.com Vol. 2, Issue 3, May-Jun 2012, pp.1313-1319 1313 I P a g e "Removal of Heart Sound from Lung Sound using LabVIEW 8.6"

[3] B. Widrow, J. Glover, JR., John McCool, John Kaunitz, Charles Williams, Robert Hearn, James Ziedler, Eugene Dong, JR. and Robert Goodlin, " Adaptive Noise Cancelling: Principles and Applications". Proceedings of the IEEE, VOL.63, NO.12 December 1975.

[4] Mohamed A. Abdulmagid*, Dean J. Krusienski, Siddharth Pal, and William K. Jenkins\#, "PRINCIPLES OF ADAPTIVE NOISE CANCELING" Department of Electrical \& Computer Engineering, The Pennsylvania State University, University Park, PA 16802.

[5] Simon Haykin, “Adaptive Filter Theory”, Prentice Hall, $4^{\text {th }}$ edition. Ying He, et. al."The Applications and Simulation of Adaptive Filter in Noise Cancelling", 2008 International Conference on Computer Science and Software Engineering, 2008, Vol.4, Page(s): 1 - 4

[6] Yuu-Seng Lau, Zahir M. Hussian and Richard Harris, "Performance of Adaptive Filtering Algorithms: A Com Study", Australian Telecommunications, Networks and Applications Conference (ATNAC), Melbourne, 2003

[7] E. Eweda, "Analysis and design of a signed regressor LMS algorithm for stationary and non stationary adaptive filterinh with correlated Gaussian data," IEEE Transations on Circuits and Systems, Vol. 37, No.11, pp.1367-1374, 1990

[8] S. Koike, "Analysis of adaptive filters using normalized signed regressor LMS algorithm," IEEE Trans. Signal Process., vol. 47 , no. 10 ,

[9] Glenn E, Johnson, Robert A Murir, Joseph N. Scanlan, William M. steedly" Practical Comparison of Adaptive filter Algorithm " The Analytic Science Corporation (TASC), PP-5259-263, (1998).

[10] Cao Yali, "The Application of LMS Algorithm in Adaptive Filter", Chinese Journal of Scientific Instrument, vol. 23, pp.54-60, May (2005)

[11] Farhang-Boroujeny, B., "Adaptive Filters- Theory and applications", John Wiley and Sons, Chichester, UK, 1998.

[12] Ondracka J., Oravec R., Kadlec J.,Cocherová E., "Simulation Of RLS And LMS Algorithms For Adaptive Noise Cancellation In MATLAB, Department Of Radio electronics FEI STU Bratislava, Slovak Republic UTLA, CAS Praha, Czech Republic.

[13] Soni Changlani \& M.K.Gupta, "Simulation of LMS Noise Canceller Using Simulink", International Journal On Emerging Technologies, 2011, ISSN: 0975-8364, pp 50-52.

[14] Ying He, Hong He, LiLi, YiWu and Hongyan Pan"The Applications and Simulation of Adaptive Filter in Noise Cancelling." International conference on computer Science and Software Engineering-2008.

[15] Raj Kumar Thenu \& S.K. Agarwal , "Hardware Implementation Of Adaptive Algorithms for Noise Cancellation", International Conference On Network Communication And Computer, 2011, pp 553-557.

[16] Raj Kumar Thenu \& S.K. Agarwal , "Simulation and Performance Analysis Of Adaptive Filter In Noise Cancellation", International Journal of Engineering science And Technology, Vol. 2(9), 2010, pp4373-4378.

[17] Soni Changlani \& Dr. M. K .Gupta, "The applications And Simulation of Adaptive Filter In Speech Enhancement", International Journal of Computer Engineering And ArchetectureVol. 1, No. 1, June 2011, pp95-101.

[18] Ferdouse L., Akhter N., Nipa T. H and Jaigirdar F. T., ,Simulation and Performance Analysis of Adaptive Filtering Algorithms in Noise Cancellatione, IJCSI International Journal of Computer Science Issues, Vol. 8, Issue 1, January 2011.

[19] Lau Y. S., Hossain Z. M., and Harris R., "Performance of Adaptive Filtering Algorithms: A Comparative Study", Proceedings of the Australian Telecommunications, Networks and Applications Conference (ATNAC), Melbourne, 2003.
[20] V.R.Vijaykumar, P.T.Vanathi \& P. Kangasapabathy, "Modified Adaptive Filtering Algorithm For Noise Cancellation In Speech signals", Electronics And Electrical Engineering, Elektronika IR Elektrotechnika,ISSN1392-1215,No. 2(74),2007,pp17-20.

\section{BIOGRAPHIES}

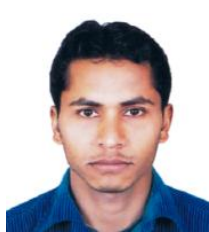

Naveen Dewangan obtained his Bachelors Degree BE (Hons.) in Electronics and Telecommunication, in 2011 and presently doing M. Tech. in Instrumentation and Control from Bhilai Institute of Technology, Durg, India. He is currently working with Shri Shankaracharya Group of Institutions (Technical Campus) Bhilai, Chhattisgarh, India as Assistant Professor in Electronics and Instrumentation Engineering Department. He had 3 years of teaching experience. His areas of interest are Adaptive Filter and its applications, Programmable logic Controller and its Application.

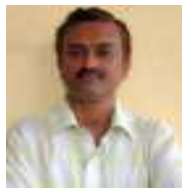

Mr. R. M. Potdar, (Phd Scholar) received B.E. Electronics, M. Tech. (Hons) Instrumentation \& Control. His interests are in Signal Processing, Neural Network \& Fuzzy Logic System Design. His specialization subjects are Adaptive Control System, Optimal Control System, Control System Design, Satellite communication and Optical Communication. He has published the papers in 8 international journals and 4 national journals and also attended 2 international conference and 14 national conferences. Also he is having Life Membership of Indian Society of Technical Education, India (ISTE).

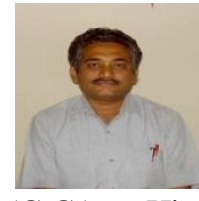

Dr. Mekhram Meshram received B.E. Electronics, ME Microwave Radar. He is having total teaching experiences of 24 year and presently he is working with government engineering college Bilaspur (C.G.). His interests are in Development and Characterization of Ferrite Based Microwave Absorber, Investigation on Channel capacity of cellular communication network. His field of specialization in Electronics and Telecommunication. He has published more than 22 research papers in the various international journals

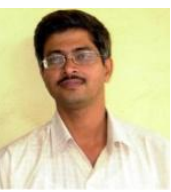

Dr. Ramesh Kumar received B E Computer Science, M. Tech (CSE). He is having total teaching experiences of 20 year and presently working with Bhilai Institute of Technology, Durg (Chhattisgarh) as a professor in department of Computer Science and Engineering. His interests are in development of a simulator for micro-optic-electro- mechanical system. His field of specialization in computer science and engineering. $\mathrm{He}$ has published the papers in 10 international journals and 5 national journals and also attended 5 international conference and 05 national conferences. He has published 42 research papers. Also he is having Life Membership of Indian Society of Technical Education, India (ISTE). 